\title{
Does plain radiography still have a role in cases of fish bone ingestion in emergency rooms? A retrospective analysis
}

\author{
Tzu-Chi Wu ${ }^{1,2}$ (D) Pin-Wen Huang ${ }^{1} \cdot$ Chun-Bin Tung ${ }^{1}$ \\ Received: 26 September 2020 / Accepted: 16 December 2020 / Published online: 27 January 2021 \\ (C) The Author(s) 2021, corrected publication 2021
}

\begin{abstract}
Background Fish bones are the most common aerodigestive foreign bodies found in adults. Most cases of fish bone impaction improve after primary management by emergency physicians with a mirror laryngoscopy using a tongue depressor, before otolaryngologists perform a fiberoptic nasendoscopy. A computed tomography scan usually follows to determine the next step. Studies have recently been concerned about overdoses of radiation from computed tomography. However, clear algorithms remain unavailable for fish bone ingestion management to date.

Methods A retrospective review was conducted on 180 patients who visited the emergency department with complaints of fish bone impaction between January 2017 and January 2019.

Results A total of $81.6 \%$ of patients with fish bone impaction got symptomatic relief after primary management by emergency physicians and otolaryngologists. Out of 180 patients, 33 (18.3\%) needed an endoscopic procedure due to persistent symptoms. Only one $(0.56 \%)$ required an operation due to perforation. In the group failing primary management, the plain radiography of eight patients showed a positive finding and an esophagogastroscopy was done to remove the fish bones.

Conclusion Lateral neck radiography is still beneficial to patients with fish bone ingestion failure from primary management. Positive lateral soft tissue radiography in cases with persistent symptoms post primary management may directly suggest esophagogastroscopy without confirmation from a computed tomography, unless complications are suspected. For patients aged below 40 , following up on their conditions after post management radiography shows negative results may increase their safety.
\end{abstract}

Keywords Fish bone $\cdot$ Foreign body $\cdot$ Lateral neck radiography $\cdot$ Radiography in emergency care

\section{Introduction}

Foreign body impaction is a common reason for emergency room visits. A direct visualization of the fishbone is the best diagnostic method and can also resolve patient anxiety. On one hand, plain lateral neck radiography makes locating fish bones difficult, because not all of them are radio opaque. On the other hand, computed tomography is effective at identifying impacted esophageal fish bones with high sensitivity. We cannot exclude fish bone impaction due to minimal falsenegative rates, especially when symptoms persist. Therefore,

Tzu-Chi Wu

j10062008@hotmail.com

1 Department of Emergency Medicine, Show Chwan Memorial Hospital, Changhua, Taiwan

2 Graduate Institute of Technology Management, National Chung Hsing University, Taichung, Taiwan endoscopic intervention plays a definite role in patients with persistent symptoms or whose computed tomography scan is positive. There have so far not been any clear algorithms available for fish bone impaction diagnosis and management. Our study fills this gap by reviewing cases of fish bone impaction and proposing a management protocol to ensure an optimum outcome for patients.

\section{Methods}

A retrospective chart review was conducted on 180 patients who complained of fish bone impaction in Taiwan's Show Chwan Memorial Hospital between January 2017 and 2019. Patients with suspected fish bones in their throats underwent a mirror laryngoscopy with the use of a tongue depressor, followed by a fiberoptic nasendoscopy performed by emergency physicians, then otolaryngologists. A lateral neck radiography was also done. After primary management, patients 
may be discharged if the foreign body is removed, or if there is symptomatic relief regardless of removal of a foreign body. Failing primary management means symptoms persist post both emergency physician and otolaryngologist management. If symptoms persisted, an esophagogastroscopy intervention was arranged (Fig. 1). We used the Mann-Whitney $U$ test and the Fisher exact test. All statistical analyses were done using the IBM SPSS ver. 20.0. The statistical significance threshold was set at $<0.05$ (two-sided test).

\section{Results}

Between January 2017 and January 2019, 147 (81.6\%) out of 180 total cases of fish bone foreign body ingestion gained symptomatic relief after the oral cavity or laryngopharynx was examined by emergency physicians or otolaryngologists. Thirty-three (18.3\%) patients needed an endoscopic procedure due to persistent symptoms. Only one $(0.56 \%)$ required an operation due to perforation as the foreign body had been in the patient's system for over 3 days. In this case, a computed tomography was performed instead.

Among the $33(18.3 \%)$ patients whose symptoms persisted post primary management were 14 men, 19 women, 8 patients below 40 years old, and 25 patients above 40 . The mean age was 54.1 years $(\mathrm{SD}, \pm 16.9)$ and body mass index was 24.1 $(\mathrm{SD}, \pm 3.94)$ (Table 1$)$. Out of all the patients $(N=33), 8$ $(24.2 \%)$ showed a positive finding from plain radiography, and $100 \%$ of patients had a fish bone removed via esophagogastroscopy (Fig. 2). The age of the group that needed an esophagogastroscopy procedure was higher than that of the group which had fish bones removed post primary management $(54.18 / 42.25, P=0.012)$. Additionally, positive findings of lateral neck radiography have a higher percentage (24.2\%) in the esophagogastroscopy procedure group $(P=$ 0.035 ) (Table 1).

Foreign bodies were successfully removed by esophagogastroscopy in 19 cases, which is $10.5 \%$ of the total number of patients. In this sample $(N=19)$, there were 9 men and 10 women, 2 patients were younger than 40 years of age, and 17 were older. Their mean age was $57.4(\mathrm{SD}, \pm 13.5)$ and body mass index (BMI) was $24.3(\mathrm{SD}, \pm 4.04)$. The fish bone was located in the vallecula or pyriform sinus of 5 patients, in the upper third of the esophagus of $13(68.4 \%)$ patients, and in the middle third of the esophagus in $1(5.2 \%)$ patient. The average length of the fish bone was $2.05 \mathrm{~cm}$. The timing of endoscopic intervention was $64.1 \mathrm{~min}$ on average, after the patient arrived at the emergency department. Moreover, fish bones were more commonly found by esophagogastroscopy in cases where patients were above 40 years old $(P=0.047)$ and in patients with positive findings on lateral neck radiography $(P=0.01)$ (Table 2$)$. The difference was significant.

\section{Discussion}

Up to $80 \%$ of patients can gain symptomatic relief after primary management by emergency physicians and the
Fig. 1 Fish bone foreign body management algorithm
Patients with symptoms of fish bone ingestion $(\mathrm{N}=180)$

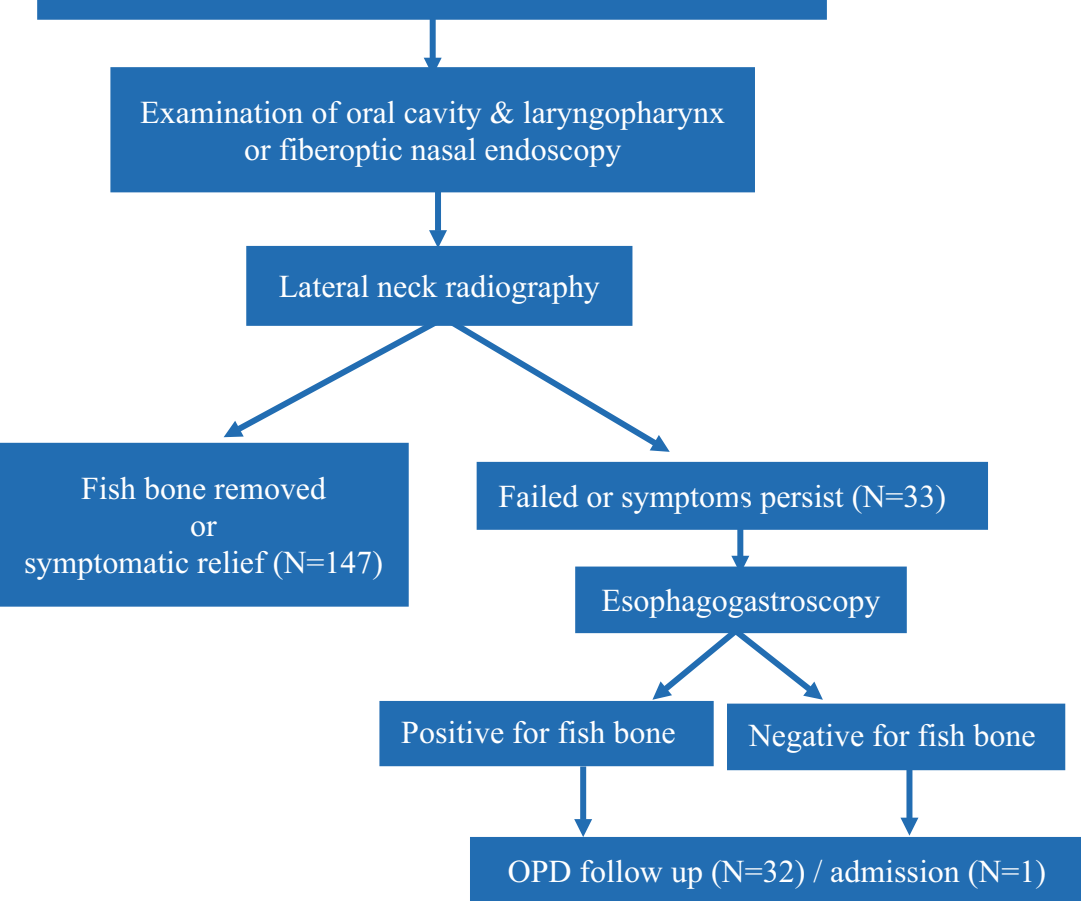


Table 1 Attributes of patients treated during primary management vs patients needing esophagogastroscopy

\begin{tabular}{llll}
\hline & $\begin{array}{l}\text { Removed by mirror } \\
\text { laryngoscopy/fiberoptic nasendoscopy }\end{array}$ & Esophagogastroscopy & $p$ value \\
\hline Number & $147(81.6 \%)$ & $33(18.3 \%)$ & \\
Age & $42.25 \pm 23.93$ & $54.18 \pm 16.91$ & 0.012 \\
Age $\leq 40$ & $65(44.3 \%)$ & $8(24.2 \%)$ & \\
Age $>40$ & $82(55.7 \%)$ & $25(75.8 \%)$ & 0.035 \\
Plain radiography positive & $13(8.8 \%)$ & $8(24.2 \%)$ & 0.035 \\
BMI & $23.04 \pm 5.29$ & $24.10 \pm 3.94$ & 0.406 \\
\hline
\end{tabular}

otolaryngologists. The most common impaction site of a fish bone is the oropharynx and oral cavity, followed by the esophagus. Within the esophagus, the upper third of the esophagus is the most common location. This may be due to the first physiological narrowing caused by the juncture with the pharynx. The risk of esophageal impaction needing endoscopic intervention increases with age. In our analysis, the risk of needing endoscopic intervention increases by 1.039 times for every 1 year of age, $P=0.024$; the difference was significant. The BMI made no difference between the two groups needing further management or not.

Oropharynx/hypopharynx are more common locations, but plain radiography is less reliable in this area due to the high soft tissue and bone density over the suprahyoid area. Overall, the positive rate of lateral neck radiography was $11.6 \%$. In a retrospective study of 698 cases with fish bones in the oropharynx [1], only $23(10 \%)$ cases were reported by the radiologist as being positive for the presence of a fish bone. This result suggested that lateral soft tissue radiography is not beneficial in cases with a suspected fish bone when fiberoptic nasendoscope is available.
In most situations, a computed tomography scan is arranged when there is a lack of findings during the fiberoptic nasendoscopy if symptoms persisted due to high sensitivity and specificity. Thus, a high percentage of those who have persistent symptoms require further intervention. According to $\mathrm{Kim}$ et al. [2], 66 (75\%) out of 88 patients with a fish bone in their esophagus underwent non-contrast-computed tomography because the foreign body was not removed through a laryngoscope. This suggests that up to $75 \%$ of patients need esophagogastroscopy if symptoms persist post laryngoscopic intervention. In our study, $19(57.6 \%)$ of the 33 cases had the fish bone removed by esophagogastroscopy, excluding those with only esophageal abrasions, bleeding, or if the fish bone slipped out to the stomach.

Studies have recently discussed that many patients, especially younger ones, undergo unnecessary imaging. In a study with 286 total cases of fish bone foreign body ingestion [2], the positive rate was higher in 88 patients aged over 40 years with non-contrast-computed tomography; younger patients below 40 are far more likely to be impacted in the oropharynx. According to Klein [3], patients below 40 years old examined within $24 \mathrm{~h}$ from ingestion show little diagnostic value from
Fig. 2 Failure after primary management

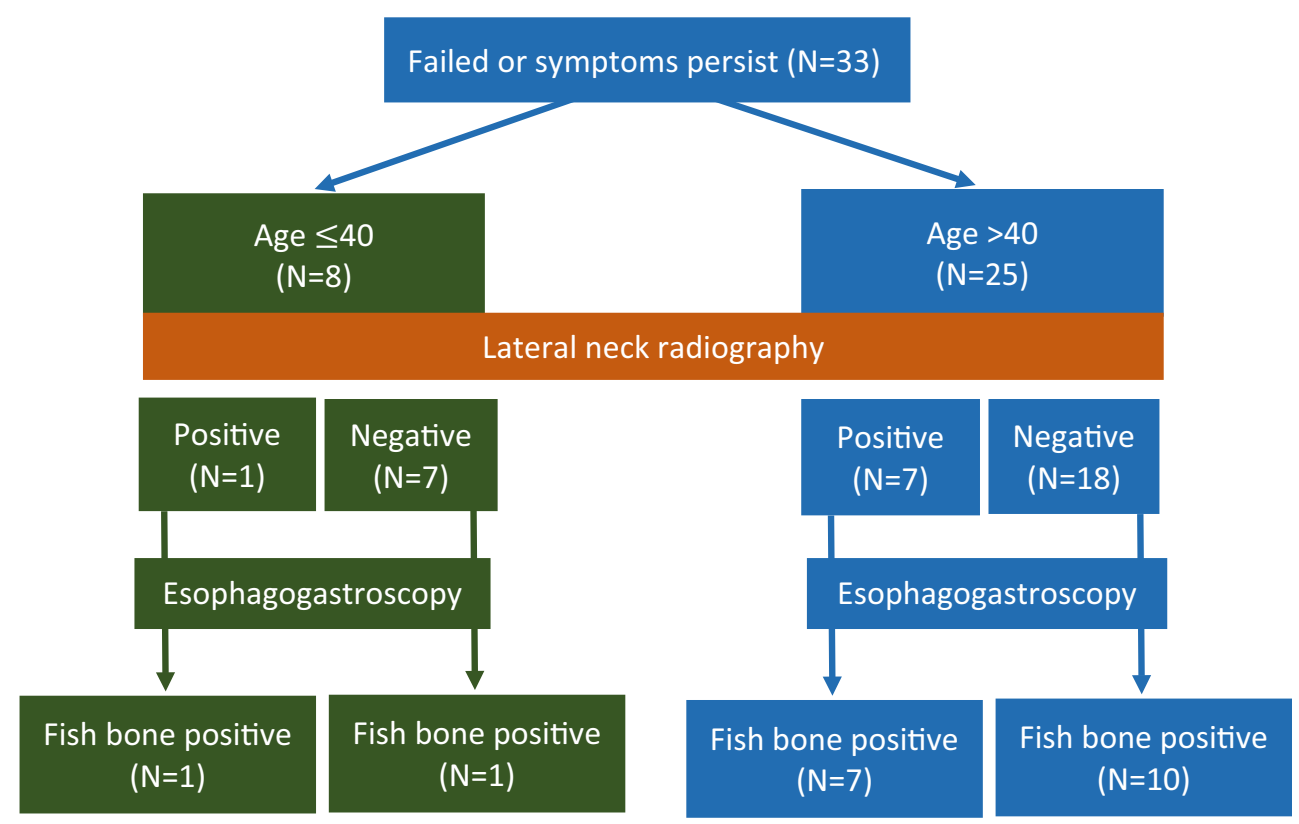


Table 2 Attributes of patients who underwent esophagogastroscopy and fish bone location

\begin{tabular}{llll}
\hline Esophagogastroscopy & $\begin{array}{l}\text { Positive with fish bone } \\
(N=19)\end{array}$ & $\begin{array}{l}\text { Negative with fish bone } \\
(N=14)\end{array}$ & $p$-value \\
\hline Sex & Male $9(47.4 \%)$ & $\begin{array}{l}\text { Male 5 }(35.7 \%) \\
\text { Female 9 (64.3\%) }\end{array}$ & \\
Age & Female 10 $(52.6 \%)$ & $49.79 \pm 20.33$ & 0.209 \\
Age $\leq 40$ & $57.42 \pm 13.56$ & $6(42.9 \%)$ & 0.047 \\
Age $>40$ & $2(10.6 \%)$ & $8(57.1 \%)$ & 0.814 \\
BMI & $17(89.4 \%)$ & $23.84 \pm 4.17$ & 0.010 \\
Plain radiography & $24.28 \pm 4.04$ & & \\
positive & & $0(0.0 \%)$ & $14(100 \%)$ \\
negative & $8(42.1 \%)$ & & \\
Fish bone location & $11(57.9 \%)$ & & \\
& Vallecula / Pyriform sinus $(N=5)$ & & \\
& Upper third of the esophagus $(N=13)$ & & \\
& Middle third of the esophagus $(N=1)$ & & \\
\end{tabular}

imaging due to the low probability of esophageal fish bones. Many young patients undergo unnecessary computed tomography studies. Therefore, some have suggested that patients younger than 40 , and visiting the emergency department less than $24 \mathrm{~h}$ after the fish bone ingestion, can be followed up after primary management without computed tomography.

Plain radiography has a much lower radiation dose than computed tomography scans. Radiation exposure is also an important concern, especially in young patients. According to most studies, it is not beneficial before primary management.
Thus, it may have a role before the computed tomography scan or discharge in some conditions.

In the group that failed the post primary management, eight patients showed a positive finding in their plain radiography, and all of them underwent esophagogastroscopy to remove the fish bone (Fig. 2). This may suggest that if the lateral soft tissue radiography is positive post primary management, an esophagogastroscopy can be done without confirming the results by computed tomography. Moreover, up to $89 \%$ of cases in the group younger than 40 years old $(N=73)$ were resolved
Fig. 3 New management algorithm of fish bone foreign body

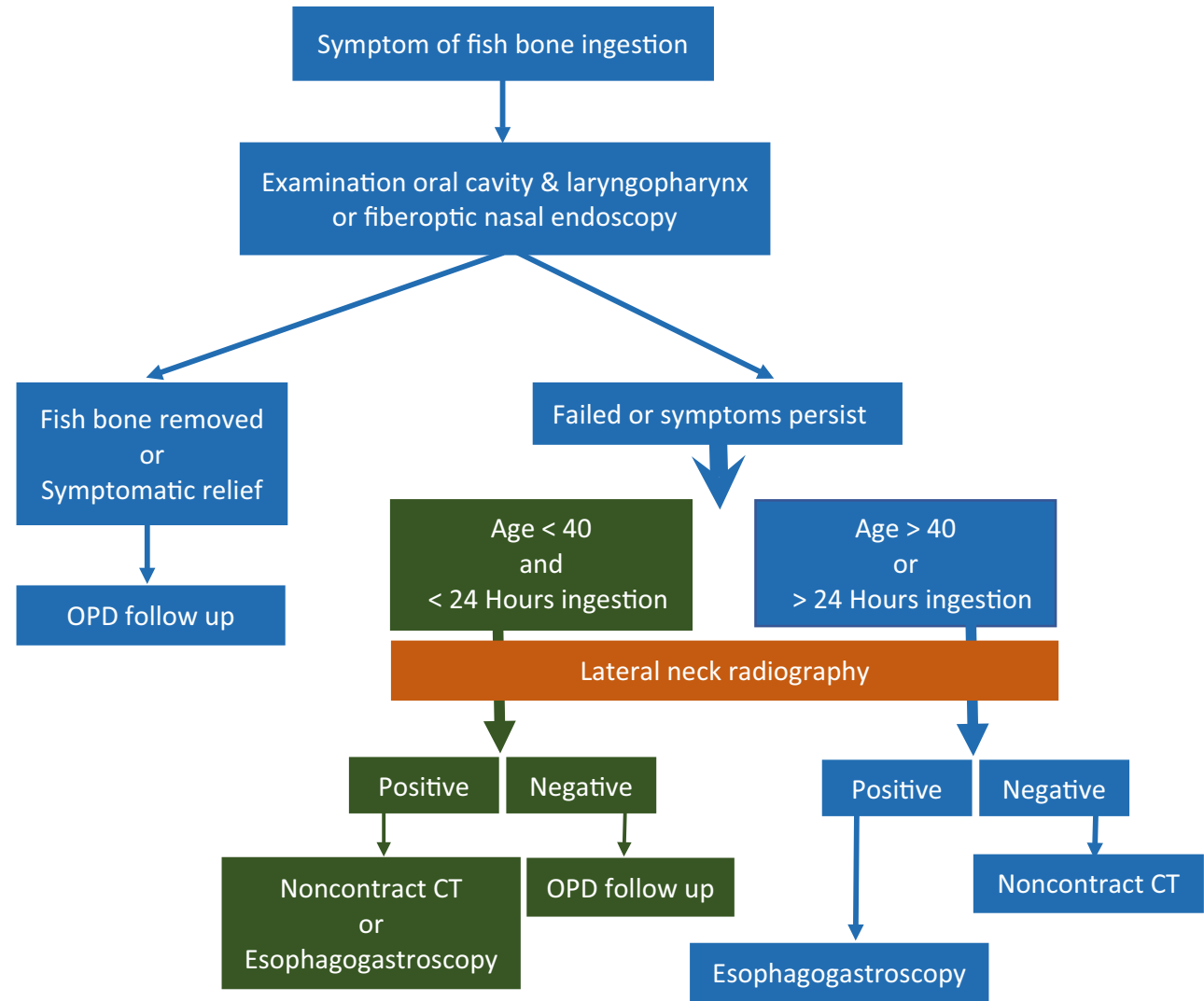


post primary management, while $8(11 \%)$ need further management. Only two showed positive findings of fish bones by esophagogastroscopy. This suggests that it is safe to discharge patients with fish bone impaction according to the management algorithm by Klein [3]. However, one in two patients younger than 40 years showed positive findings of fish bones. If we check the plain radiography before discharge, one may directly arrange esophagogastroscopy without observation or a computed tomography scan if symptoms persist. Further, a computed tomography scan is unnecessary for the group aged above 40 whose plain radiography came out positive for the presence of fish bones, unless complications, such as abscess formation or perforation, are suspected (Fig. 3).

The timing of endoscopic intervention is associated with risks of aspiration and perforation. According to the European Society of Gastrointestinal Endoscopy guidelines, sharppointed objects such as fish bone foreign bodies are considered an emergency condition and should preferably be removed within $6 \mathrm{~h}$ at most. Esophageal foreign bodies without complete obstruction are considered an urgent condition that requires therapeutic esophagogastroduodenoscopy to be performed within $24 \mathrm{~h} \mathrm{[4]}$. Therefore, in situations where a computed tomography scan is not available within $24 \mathrm{~h}$, lateral soft tissue radiography may improve the safety of patients and clear the pathway.

This study has several limitations. Primarily, the size of the study was small when compared with other studies. Second, it was a retrospective study which carries its own bias and weaknesses.

Up to $80 \%$ of the patients get symptomatic relief after management by emergency department physicians and then otolaryngologists. If the examination is unremarkable and symptoms persist, lateral neck radiography still plays a role. Plain radiography, showing positive in cases where symptoms persist post primary management, may be directly followed by an esophagogastroscopy without confirming a computed tomography, unless complications are suspected. For patients younger than 40 years old, outpatient department follow-up post negative of neck radiography may improve the safety of patients.

\section{Compliance with ethical standards}

Conflict of interest The authors declare that they have no conflict of interest.

Open Access This article is licensed under a Creative Commons Attribution 4.0 International License, which permits use, sharing, adaptation, distribution and reproduction in any medium or format, as long as you give appropriate credit to the original author(s) and the source, provide a link to the Creative Commons licence, and indicate if changes were made. The images or other third party material in this article are included in the article's Creative Commons licence, unless indicated otherwise in a credit line to the material. If material is not included in the article's Creative Commons licence and your intended use is not permitted by statutory regulation or exceeds the permitted use, you will need to obtain permission directly from the copyright holder. To view a copy of this licence, visit http://creativecommons.org/licenses/by/4.0/.

\section{References}

1. Sanei-Moghaddam A, Sanei-Moghaddam A, Kahrobaei S (2015) Iranian lateral soft tissue X-ray for patients with suspected fishbone in oropharynx, a thing in the past. J Otorhinolaryngol 27(6):Serial No.83

2. Jin Pyeong Kim OJK, Kim HSSRB, Kim JH, Woo SH (2015) Analysis of clinical feature and management of fish bone ingestion of upper gastrointestinal tract. Clin Exp Otorhinolaryngol 8(3):261267

3. Klein A, Ovnat-Tamir S, Marom T, Gluck O, Rabinovics N, Shemesh S (2019) Fish bone foreign body: the role of imaging. Int Arch Otorhinolaryngol 23:110-115

4. Michael Birk PB, Deprez PH (2016) Removal of foreign bodies in the upper gastrointestinal tract in adults: European Society of Gastrointestinal Endoscopy (ESGE) Clinical Guideline. Endoscopy $48: 1-8$

Publisher's note Springer Nature remains neutral with regard to jurisdictional claims in published maps and institutional affiliations. 